\title{
Validity of Quantitative Lymphoscintigraphy as a Lymphedema Assessment Tool for Patients With Breast Cancer
}

\author{
Ji-Na Yoo, $\mathrm{MD}^{1}$, Youn-Soo Cheong, $\mathrm{MD}^{1}$, Yu-Sun Min, $\mathrm{MD}^{2}$, \\ Sang-Woo Lee, $\mathrm{MD}^{3}$, Ho Yong Park, $\mathrm{MD}^{4}$, Tae-Du Jung, $\mathrm{MD}^{1,2}$ \\ ${ }^{1}$ Department of Rehabilitation Medicine, Kyungpook National University College of Medicine, Daegu; \\ ${ }^{2}$ Department of Rehabilitation Medicine, Kyungpook National University Hospital, Daegu; \\ ${ }^{3}$ Department of Nuclear Medicine, Kyungpook National University Medical Center, Daegu; \\ ${ }^{4}$ Department of Surgery, Breast Cancer Center, Kyungpook National University Medical Center, Daegu, Korea
}

Objective To evaluate the validity of quantitative lymphoscintigraphy as a useful lymphedema assessment tool for patients with breast cancer surgery including axillary lymph node dissection (ALND).

Methods We recruited 72 patients with lymphedema after breast cancer surgery that included ALND. Circumferences in their upper limbs were measured in five areas: $15 \mathrm{~cm}$ proximal to the lateral epicondyle (LE), the elbow, $10 \mathrm{~cm}$ distal to the LE, the wrist, and the metacarpophalangeal joint. Then, maximal circumference difference (MCD) was calculated by subtracting the unaffected side from the affected side. Quantitative asymmetry indices (QAI) were defined as the radiopharmaceutical uptake ratios of the affected side to the unaffected side. Patients were divided into 3 groups by qualitative lymphoscintigraphic patterns: normal, decreased function, and obstruction.

Results The MCD was highest in the qualitative obstruction (2.76 \pm 2.48$)$ pattern with significant differences from the normal $(0.69 \pm 0.78)$ and decreased function $(1.65 \pm 1.17)$ patterns. The QAIs of the axillary LNs showed significant differences among the normal $(0.82 \pm 0.29)$, decreased function $(0.42 \pm 0.41)$, and obstruction $(0.18 \pm 0.16)$ patterns. As the QAI of the axillary LN increased, the MCD decreased. The QAIs of the upper limbs were significantly higher in the obstruction $(3.12 \pm 3.07)$ pattern compared with the normal $(1.15 \pm 0.10)$ and decreased function $(0.79 \pm 0.30)$ patterns.

Conclusion Quantitative lymphoscintigraphic analysis is well correlated with both commonly used qualitative lymphoscintigraphic analysis and circumference differences in the upper limbs of patients with breast cancer surgery with ALND. Quantitative lymphoscintigraphy may be a good alternative assessment tool for diagnosing lymphedema after breast cancer surgery with ALND.

Keywords Breast cancer, Lymph node dissection, Upper extremity, Lymphedema, Lymphoscintigraphy

(c) This is an open-access article distributed under the terms of the Creative Commons Attribution Non-Commercial License (http://creativecommons.org/ licenses/by-nc/4.0) which permits unrestricted noncommercial use, distribution, and reproduction in any medium, provided the original work is properly cited. Copyright () 2015 by Korean Academy of Rehabilitation Medicine 


\section{INTRODUCTION}

Secondary lymphedema during the treatment of malignancies is common, especially in breast cancer patients who are treated with axillary lymph node dissection (ALND) [1]. Previous studies have reported the incidence of secondary lymphedema after breast cancer treatment to range from $2 \%$ to $83 \%$, but the incidence is generally accepted as being approximately $30 \%$ in patients treated with axillary surgery $[2,3]$. Nevertheless, patients with lymphedema will still suffer without appropriate interventions if their primary physician or even surgeon lacks a complete understanding of its therapeutic importance [4]. Some previous studies showed that even mild lymphedema could be problematic, both cosmetically and psychologically, and that it could decrease the quality of life of patients with breast cancer surgery with ALND $[5,6]$. Therefore, early diagnosis of lymphedema for early intervention with an objective measurement tool is important [7-9].

For objective assessment of lymphedema and its severity, methods such as measuring limb circumference and/or volume, performing tissue tonometry, and/or conducting water displacement tests have been used [10]. Of these, measuring limb circumference is the most commonly used diagnostic tool for lymphedema because it is simple and convenient, although it has been controversial. Recently, lymphoscintigraphy has been widely considered to be the most appropriate tool for providing a more objective evaluation of lymphedema [11-14].

In contrast with other measurement tools that only reflect lymphedema volume or soft tissue composition, lymphoscintigraphy offers an objective and reliable approach to diagnosing and specifically characterizing the severity of lymphedema with visualization of regional lymph nodes (LNs), lymphatic channels, collateral lymphatic channels, interrupted vascular structures, and the deep lymphatic nodes [15]. Until recently, lymphoscintigraphic interpretations have generally been qualitative. These qualitative findings include asymmetric uptake patterns of radiopharmaceuticals in the proximal LNs, the main lymphatic vessel, the presence of radiopharmaceutical uptake in collateral lymphatic vessels, and abnormal dermal backflow. Hwang et al. [16] used a qualitative lymphoscintigraphic interpretation as a prognostic tool for breast cancer-related lymphedema patients, and the lymphoscintigraphic findings of the main lymphatic vessels without collateral lymphatic vessels were the best predictors of a positive response to lymphedema treatment. A number of studies have assessed the value of lymphoscintigraphy in gynecological cancer-related lower limb lymphedema, which is a common complication after treatment $[15,17]$. Because Stanton et al. [18] used the drainage rate constant $(\mathrm{k})$ of lymphoscintigraphy to quantitatively diagnose ipsilesional hand lymphedema following breast cancer treatment, we decided that quantitative lymphoscintigraphic findings could provide more objective data for treating patients who have lymphedema after breast cancer surgery with ALND.

The aim of this study was to evaluate whether quantitative and qualitative lymphoscintigraphic analyses are correlated with each other and with circumference differences in the upper limbs of patients who have had breast cancer surgery with ALND-that is, whether quantitative analyses are a good alternative assessment tool for diagnosing lymphedema.

\section{MATERIALS AND METHODS}

\section{Subjects}

A retrospective chart review was performed for patients who were clinically diagnosed with upper limb lymphedema by post-operative onset of lymphedema symptoms in the Department of Rehabilitation Medicine between February 2011 and December 2014. Inclusion criteria for this study were 1) clinically diagnosed lymphedema with chief complaint of aching, discomfort, feeling of heaviness or tightness, and swelling, hardening, and thickening of the skin of the arm ipsilateral to the BCS site; 2) history of breast cancer surgery with ALND; 3) history of radionuclide lymphoscintigraphy for clinically diagnosed lymphedema and having already been categorized by qualitative lymphoscintigraphic patterns as normal, decreased function, or obstruction; and 4) having measurements of the differences in circumference between the affected and unaffected upper limbs. Exclusion criteria were 1) presence of certain comorbidities (current metastasis, vascular disease, active conditions such as cellulitis, or wound infections of the affected upper limb), 2) history of trauma, 3) history of surgery to the affected upper limb, and 4) dissection of only sentinel LNs. 
The present research was approved by the Kyungpook National University Hospital Institutional Review Board (No. 2015-03-041).

\section{Lymphedema assessment}

\section{Quantitative lymphoscintigraphic analysis}

The radiopharmaceutical used in this study was 185 MBq radiolabeled ${ }^{99 \mathrm{~m}} \mathrm{Tc}$ phytate. After subcutaneous injection of particles into the second interdigital spaces of the dorsum of both hands using a 25-gauge needle, patients were immediately encouraged to exercise with a rubber ball to encourage lymphatic flow in both upper limbs for 30 minutes. Follow-up planar images were taken 30 minutes, 1 hour, and 2 hours from the injection time using a dual-head gamma camera (Infinia Hawkeye; GE Medical Systems, Milwaukee, WI, USA). Data were analyzed with Xeleris software (GE Medical Systems). We used the images that were obtained at 2 hours, which were the most accurate for demonstrating the proximal lymphatics and abnormal dermal backflow even in patients with very slow lymphatic progression [19-21]. The radioscintigraphic results were interpreted by consensus of two nuclear medicine physicians who were blinded to other clinical information.

The quantitative lymphoscintigraphic analysis used the quantitative asymmetry index (QAI) for both upper limbs with reference to previous studies $[18,22]$. The regions of interest (ROIs) of both axillary LNs were drawn with circular boundaries of the same size as the radiopharmaceutical uptake areas of the unaffected axillary LNs in anticipated symmetric locations. The ROIs of both upper limb areas were drawn with rectangular boundaries that were the same size as the unaffected upper limb areas. Because of excessive collateral LN uptake at the elbow and high radioactivity from the radiopharmaceutical injection in the hand, the area of the rectangular boundary was divided into the upper arm and the forearm region to exclude the elbow and hand. At the time of analysis, we ignored the asymptomatic upper limb area, and the selected area was the upper arm or forearm region (Fig. 1). The asymmetry indices of the axillary LN and the upper limb were calculated with the radioactive ROI using the following formula, where the gamma count was the total radiopharmaceutical uptake in the ROI:

$$
\text { QAI }=\frac{\text { gamma count in ROI of affected side }}{\text { gamma count in ROI of unaffected side }}
$$

\section{Qualitative lymphoscintigraphic analysis}

Qualitative lymphoscintigraphic patterns were also described by analyzing the uptake patterns of the axillary LNs and the symptomatic areas of the upper limbs $[15,18]$.
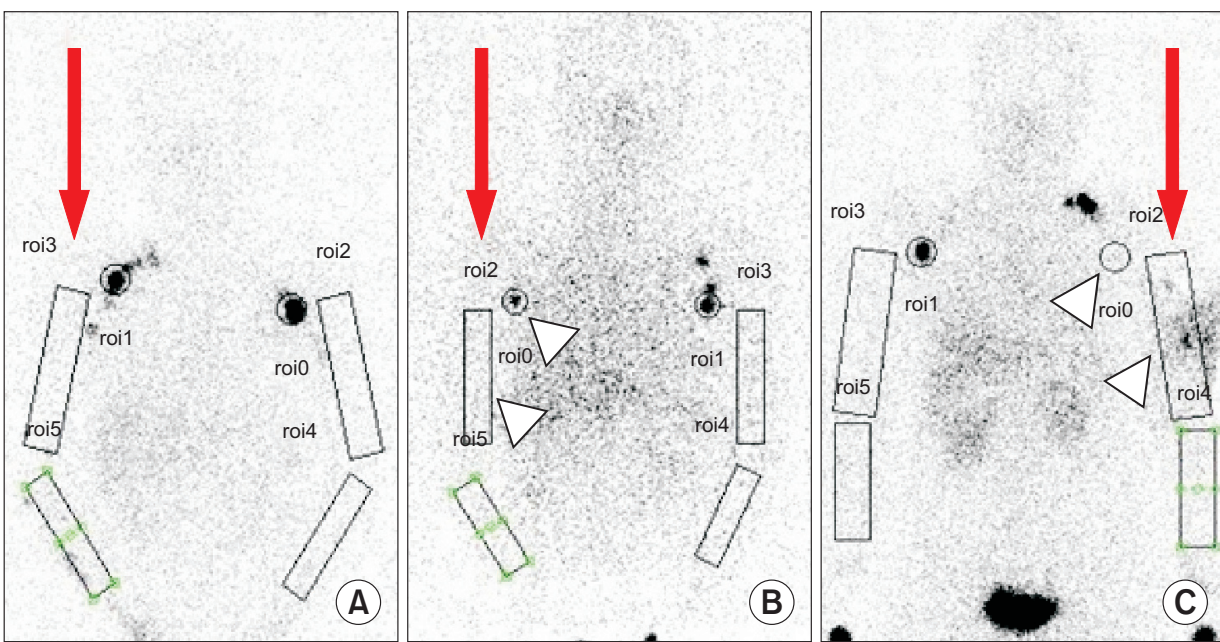

Fig. 1. Typical images of quantitative lymphoscintigraphic analysis of the upper limb of a patient with breast cancer surgery including axillary lymph node (LN) dissection. The axillary LN and symptomatic upper limb area were analyzed quantitatively on both the affected (arrow) and unaffected sides. (A) The normal pattern showed symmetric uptake of ${ }^{99 \mathrm{~m}} \mathrm{Tc}$ phytate at the axillary LN and upper limb, (B) the decreased function pattern showed decreased uptake at both the axillary LN and upper limb (arrowhead), and (C) the obstruction pattern showed little uptake at the axillary LN and highly increased uptake in the upper limb (arrowhead). 


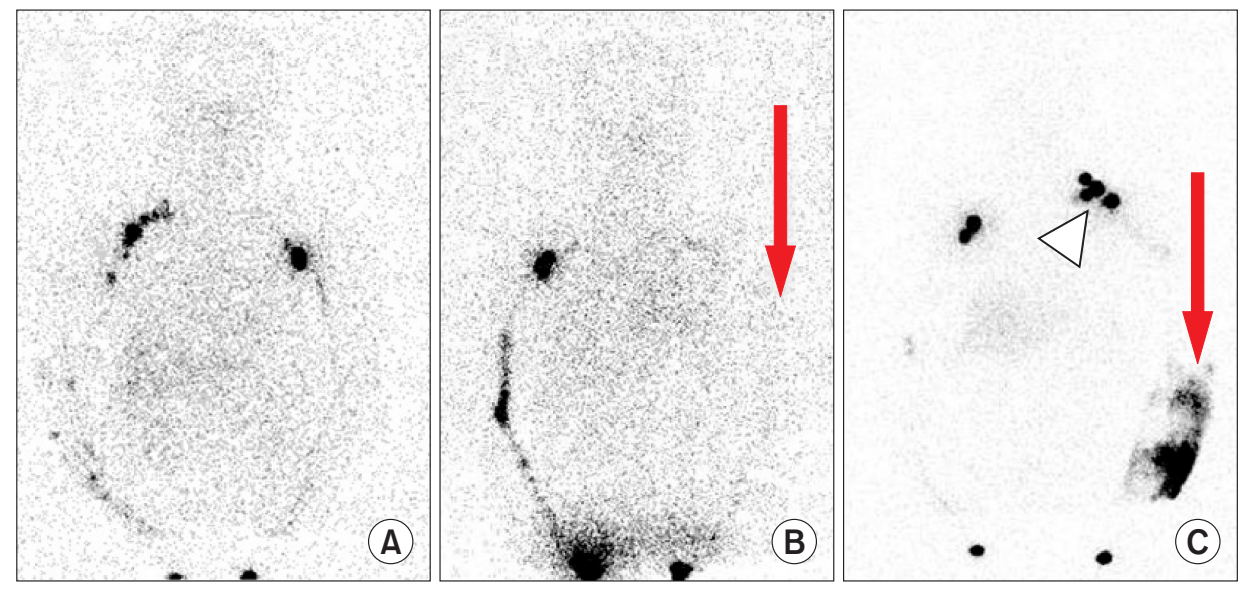

Fig. 2. Typical images of qualitative lymphoscintigraphic patterns of the upper limb of a patient with breast cancer surgery including axillary lymph node (LN) dissection. (A) The normal pattern showed symmetric visualization of the lymphatics in the limb area, (B) the decreased function pattern showed decreased visualization of lymphatics (arrowhead), and (C) the obstruction pattern showed abnormal dermal backflow (arrow) and visualization of the collateral LN (arrowhead).

Patterns were defined as follows: a normal pattern showed a normal lymphatic system with visualization of the superficial lymphatic system and a normal axillary LN. A decreased function pattern showed decreased visualization of lymphatic channels or delayed lymphatic flow ipsilateral to the breast lesion. An obstruction pattern showed abnormal dermal backflow or few or no axillary LNs (Fig. 2).

\section{Maximal circumference differences in the upper limbs}

The upper limb circumferences were estimated using a non-elastic measuring tape in five areas on the same day as the lymphoscintigraphy: 1) $15 \mathrm{~cm}$ proximal to the lateral epicondyle of the humerus; 2) the elbow, specifically, the mid-point between the medial and lateral epicondyle; 3) $10 \mathrm{~cm}$ distal to the lateral epicondyle; 4) the wrist, specifically, the mid-point of the wrist crease; and 5) the metacarpophalangeal joint $[23,24]$ (Fig. 3). To maximize reliability, estimated circumferences were recorded by calculating the average value of two measurements by one specialist physical therapist who was blinded to other clinical information. Then, the maximal circumference differences (MCDs) at the uppermost symptomatic upper limb areas were calculated [25].

\section{Statistical analysis}

All statistical analyses were conducted using SPSS software ver. 19 for Windows (IBM SPSS, Armonk, NY, USA).

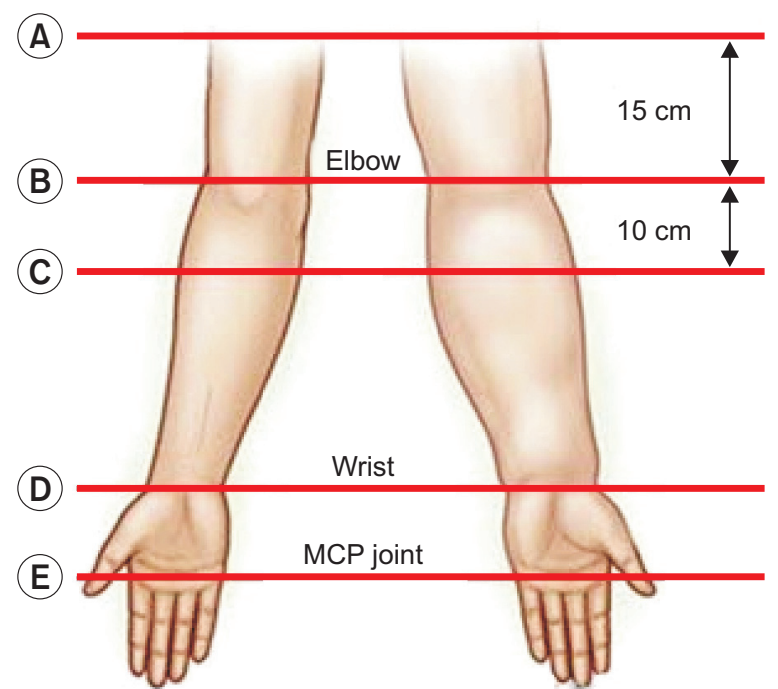

Fig. 3. Sites for measuring a patient's upper limb circumference. The five points are marked: (A) $15 \mathrm{~cm}$ proximal to the lateral epicondyle of humerus, (B) the elbow, specifically, the mid-point between the medial and lateral epicondyle, (C) $10 \mathrm{~cm}$ distal to the lateral epicondyle, (D) the wrist, specifically, the mid-point of the wrist crease, and (E) the metacarpophalangeal (MCP) joint.

To confirm the correlations between the quantitative and qualitative data, and the correlations between the qualitative data and the MCDs at the upper limbs, the Kruskal-Wallis test was used. Specifically, the $\chi^{2}$ and Kruskal-Wallis tests were used to compare qualitative lymphoscintigraphic pattern baseline characteristics. 
Pearson correlation test was used to analyze the correlations between quantitative data and MCDs of the upper limbs. Results were considered statistically significant if the $\mathrm{p}$-value was $<0.05$. Intraclass correlation coefficients (ICCs) were calculated to express the intra-rater and inter-rater reliability of the measurements. ICCs can range from 0 to 1 , with a higher value indicating better reliability. An ICC $<0.40$ is considered poor, $0.40-0.59$ is fair, $0.60-0.74$ is good, and $0.75-1.00$ is excellent.

\section{RESULTS}

General characteristics based on qualitative lymphoscintigraphic analysis

Our study comprised 72 patients with lymphedema after breast cancer surgery with ALND. All patients were women, and their mean age was $53.0 \pm 8.1$ years. The qualitative lymphoscintigraphic patterns revealed that 7 patients showed a normal pattern, 38 had a decreased function pattern, and 27 had an obstruction pattern. No statistically significant differences were found by pattern with regard to breast cancer stage, pathology, surgery type, having received radiotherapy or chemotherapy, body mass index $\geq 25$, or time after surgery (Table 1 ).
Correlation between quantitative and qualitative lymphoscintigraphic analysis

In the quantitative lymphoscintigraphic analyses, the QAIs at the axillary LNs were significantly lowest in the obstruction pattern and highest in the normal pattern among the three qualitative patterns. The decreased function pattern showed significantly higher QAIs than did the obstruction pattern and lower QAIs than in the normal pattern. At the axillary LNs, the indices were $0.82 \pm 0.29,0.42 \pm 0.41$, and $0.18 \pm 0.16$ for the normal, decreased function, and obstruction patterns, respectively.

However, in the upper limb areas, only the values for the obstruction pattern were significantly higher than the others, with the lowest values in the decreased function pattern. In the upper limb areas, the indices were $1.15 \pm 0.10,0.79 \pm 0.30$, and $3.12 \pm 3.07$ for the normal, decreased function, and obstruction patterns, respectively (Table 2, Fig. 4).

Correlation between quantitative lymphoscintigraphic analysis and maximal circumference difference

Higher QAIs of the axillary LNs showed significant inverse correlations with the MCDs in the upper limbs. The QAIs were $0.82 \pm 0.29,0.42 \pm 0.41$, and $0.18 \pm 0.16$, and the

Table 1. Characteristics of patients with lymphedema after breast cancer surgery

\begin{tabular}{|c|c|c|c|c|}
\hline Characteristic & $\begin{array}{c}\text { Normal pattern } \\
(n=7)\end{array}$ & $\begin{array}{c}\text { Decreased function } \\
\text { pattern }(n=38)\end{array}$ & $\begin{array}{c}\text { Obstruction } \\
\text { pattern }(n=27)\end{array}$ & p-value \\
\hline Age (yr) & $51.0 \pm 6.3$ & $52.3 \pm 8.4$ & $54.5 \pm 8.2$ & 0.46 \\
\hline \multicolumn{5}{|l|}{ Breast cancer stage } \\
\hline I & $0(0)$ & $1(2.6)$ & $1(3.7)$ & 0.92 \\
\hline II & $3(42.8)$ & $21(55.3)$ & $15(55.6)$ & \\
\hline III & $2(28.6)$ & $12(31.6)$ & $7(25.9)$ & \\
\hline IV & $2(28.6)$ & $4(10.5)$ & $4(14.8)$ & \\
\hline \multicolumn{5}{|l|}{ Pathology } \\
\hline Invasive ductal & $7(100)$ & $37(97.4)$ & $26(96.3)$ & 0.87 \\
\hline Invasive lobular & $0(0)$ & $1(2.6)$ & $1(3.7)$ & \\
\hline \multicolumn{5}{|l|}{ Surgery type } \\
\hline BCS & $2(28.6)$ & $14(36.8)$ & $11(40.7)$ & 0.83 \\
\hline MRM & $5(71.4)$ & $24(63.2)$ & $16(59.3)$ & \\
\hline Patients with radiotherapy & $4(57.1)$ & $22(57.9)$ & $16(59.3)$ & 0.99 \\
\hline Patients with chemotherapy & $4(57.1)$ & $20(52.6)$ & $17(62.96)$ & 0.78 \\
\hline Patients with $\mathrm{BMI} \geq 25$ & $2(28.6)$ & $10(26.3)$ & $9(33.3)$ & 0.86 \\
\hline Time after surgery (mo) & $16.7(15.4)$ & $21.6(17.8)$ & $23.3(18.8)$ & 0.29 \\
\hline
\end{tabular}

Values are presented as mean \pm standard deviation or number (\%).

BCS, breast-conserving surgery; MRM, modified radical mastectomy; BMI, body mass index. 
Table 2. Clinical and lymphoscintigraphic parameters

\begin{tabular}{lcccc}
\hline & $\begin{array}{c}\text { Normal pattern } \\
(\mathbf{n}=7)\end{array}$ & $\begin{array}{c}\text { Decreased function } \\
\text { pattern }(\mathbf{n}=\mathbf{3 8})\end{array}$ & $\begin{array}{c}\text { Obstruction } \\
\text { pattern }(\mathbf{n}=\mathbf{2 7})\end{array}$ & p-value \\
\hline QAI & & & & \\
Axillary LN & $0.8 \pm 0.29$ & $0.42 \pm 0.41$ & $0.18 \pm 0.16$ & $<0.001^{*}$ \\
$\quad$ Upper limb & $1.15 \pm 0.10$ & $0.79 \pm 0.30$ & $3.12 \pm 3.07$ & $<0.001^{*}$ \\
MCD between both upper limbs (cm) & $0.69 \pm 0.78$ & $1.65 \pm 1.17$ & $2.76 \pm 2.48$ & $0.009^{*}$ \\
\hline
\end{tabular}

Values are presented as mean \pm standard deviation.

QAI, quantitative asymmetry index; LN, lymph node; MCD, maximal circumference difference.

${ }^{*} \mathrm{p}<0.05$.

(A)

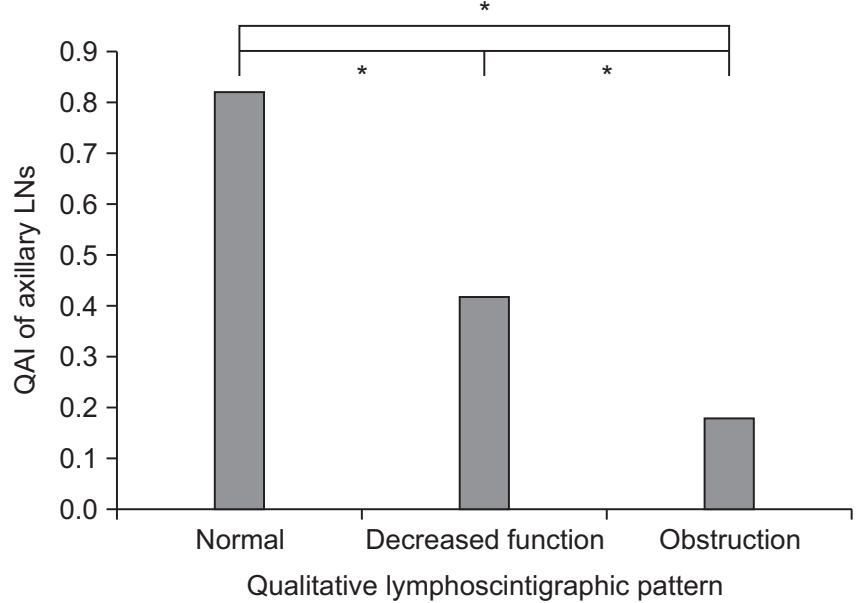

(C)

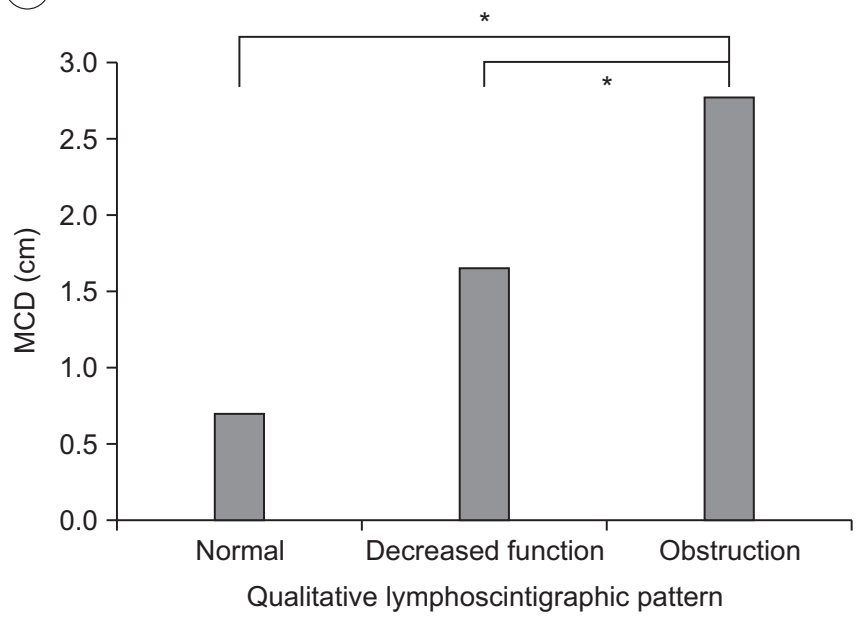

MCDs were $0.69 \pm 0.78,1.65 \pm 1.17$, and $2.76 \pm 2.48$ for the normal, decreased function, and obstruction patterns, respectively. In addition, higher QAIs for the upper limbs showed significantly higher MCDs, and QAIs that approached 1 significantly correlated with lower MCDs (Fig. 5).

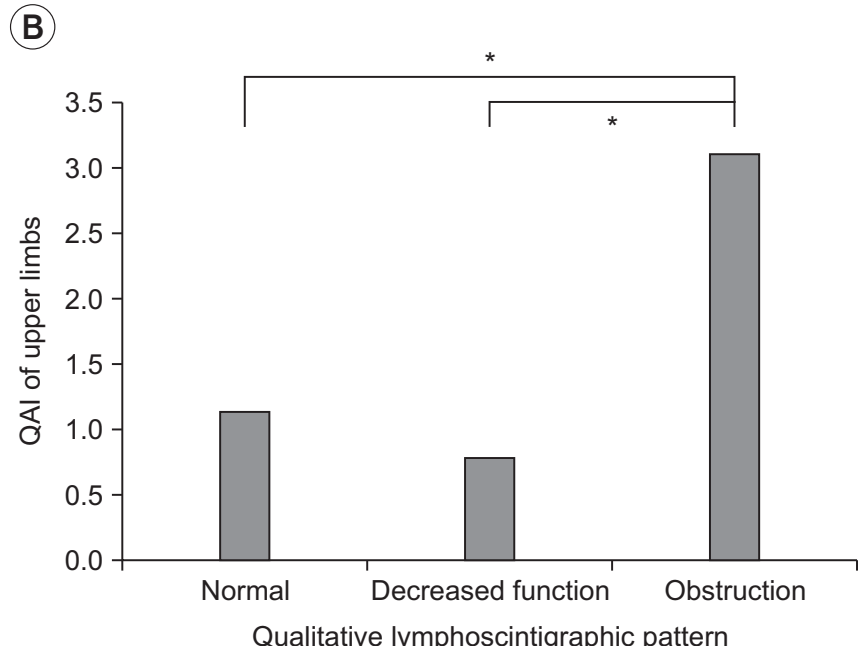

Fig. 4. Correlations among the quantitative asymmetry index (QAI) and qualitative lymphoscintigraphic patterns (A, B) and maximal circumference difference (MCD) (C). (A) Asymmetry index at the axillary lymph node (LN) showed significant differences among the three qualitative patterns, (B) QAIs at the upper limb areas in the obstruction pattern were significantly higher than those in the other patterns, and (C) the MCDs between the upper limbs in the obstruction pattern were significantly different from the other patterns $\left({ }^{*} \mathrm{p}<0.05\right)$.

\section{Correlation between qualitative lymphoscintigraphic} analysis and maximal circumference difference

MCDs in both upper limbs in the obstruction pattern were significantly higher than those in the other patterns, and MCDs in the normal pattern were lower than those for decreased function; specifically, the values were 
(A)

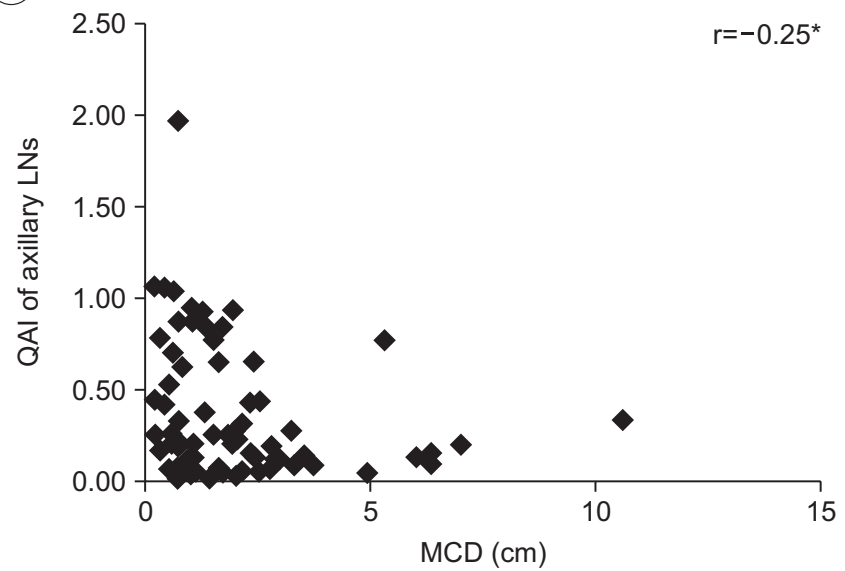

(B)

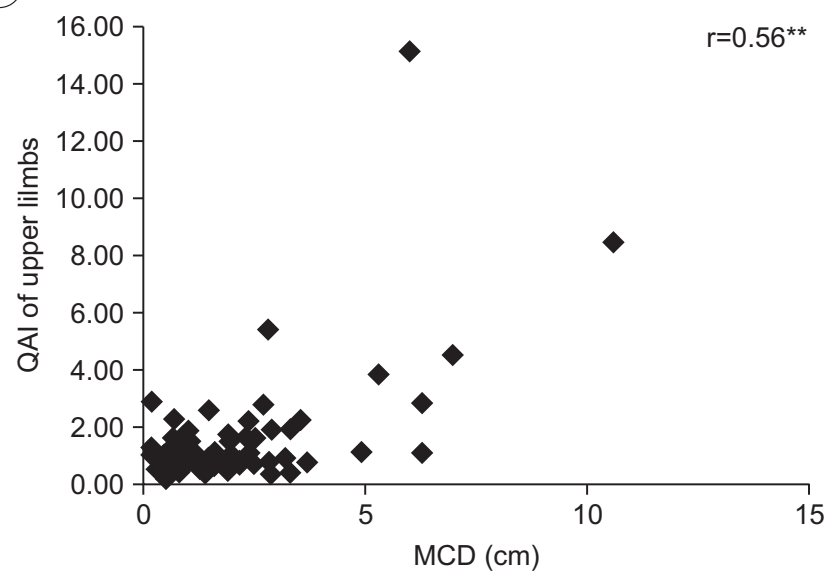

Fig. 5. Correlation between quantitative asymmetry index (QAI) and maximal circumference difference (MCD) between the upper limbs. (A) Higher QAIs axillary lymph nodes showed significant inverse correlations with the MCDs between the upper limbs and (B) higher QAIs in the upper limbs were significantly correlated with the MCDs between the upper limbs $\left({ }^{*} \mathrm{p}<0.05,{ }^{* *} \mathrm{p}<0.01\right)$.

$0.69 \pm 0.78,1.65 \pm 1.17$, and $2.76 \pm 2.48$ for the normal, decreased function, and obstruction patterns, respectively. This showed that the obstruction pattern was more highly related to the MCDs in both upper limbs compared with the other patterns (Table 2, Fig. 4).

Reliability of quantitative lymphoscintigraphic analysis The overall intra-rater reliability (ICC $=0.946)$ and interrater reliability (ICC $=0.846$ ) for lymphedema assessment in patients who had breast cancer surgery with ALND were excellent.

\section{DISCUSSION}

This is the first study to evaluate whether quantitative lymphoscintigraphic analyses correlate with qualitative lymphoscintigraphic analyses and circumference differences in breast cancer surgery patients with ALND and, consequently, whether quantitative analyses are a useful lymphedema assessment tool. Patients with a qualitative obstruction pattern had higher QAIs in their upper limbs and lower values in their axillary LNs, which reflected the decreased functioning of the normal main lymphatic flow to the proximal LN with increased abnormal collateral lymphatic flow such as abnormal dermal backflow. In the same way, MCDs were significantly correlated with higher QAIs in the upper limbs and lower QAIs in the axillary LNs. In addition, patients with the obstruction pattern showed statistically significant differences in the maximal circumferences of their upper limbs, as in previous studies [19].

In breast cancer surgery patients with ALND, disrupted lymphatic patency from the surgery results in secondary lymphedema [26]. To measure swelling of the ipsilesional upper limb, the circumference difference method was not the standard, but it is the most commonly used lymphedema assessment tool clinically $[27,28]$ because measuring circumference differences is simple and costeffective. However, there are limitations to this method, such as hand dominance, fluid shifting, and irregular hand shapes. The mean normal inter-limb difference is $3 \%$ [29]. Some shifting of fluids is inevitable because of a patient's positioning and external compression along with irregularities in hand shape. In addition, this method allows for assessing total limb volume, but it may not reflect changes in extracellular fluid volume and thus is not a specific measure of lymphedema [30].

To date, diagnosis of lymphatic dysfunction has been largely clinical, and additional assessments have only been used when the diagnosis is not completely clear [14]. However, over the past 20 years, lymphoscintigraphy has become an increasingly valuable tool and is now in widespread use around the world [19]. In fact, it is now established as the most advanced method for assessing the patency of lymphatics. It offers safety, ease of performance, and clear pre-morbid symmetry between the 
two arms with respect to both depot clearance and blood appearance rates [31]. In this study, we hypothesized that quantitative lymphoscintigraphic analyses that reflected the patency and function of lymphatics would be correlated with both widely used qualitative lymphoscintigraphic analyses and circumference differences in the upper limbs of breast cancer surgery patients with ALND.

A number of studies of lymphedema have used qualitative or quantitative lymphoscintigraphic analyses. Pecking et al. [19] found that qualitative lymphoscintigraphy that described the superficial and deep lymphatic systems, the proximal LNs, and abnormal dermal backflow reflected clinical lymphedema in the lower limbs. The presence of abnormal dermal backflow was correlated with severe lymphedema, as we also found. However, the authors of that study did not make a quantitative analysis and assessed only 60-minute images after radiopharmaceutical injections, which might not fully cover the delayed uptake of proximal LNs in lower limb lymphedema. Szuba et al. [22] revealed that a quantitatively calculated ratio of radioactivity within the affected to normal axillae was correlated with clinical swelling using the percentage of reduced edema volume in breast cancer patients. However, they found only proximal LN dysfunction, without significant lymphatic differences from the limb areas and collateral lymphatics, and they included lymphedema patients with non-operative treatment of breast cancer.

Our study included not only the proximal LNs but also the symptomatic limb areas. In the axillary LNs, both the qualitative obstruction pattern and a higher MCD were significantly correlated with a lower QAI. These findings may indicate that more severe lymphedema caused more severely impaired drainage of lymphatic fluid to the proximal LN. In this regard, both the qualitative decreased function pattern and a moderate degree of MCD were well correlated with moderately higher QAIs, and both the qualitative normal pattern and a lower MCD were also significantly correlated with a higher QAI. In contrast, in the upper limb area, both the qualitative obstruction pattern and a larger MCD were significantly correlated with a higher QAI. These results would seem to reflect increased collateral lymphatic flow in more severe lymphedema [18]. Additionally, and different from the results for the axillary LN area, the qualitative decreased function pattern showed the significantly lowest QAIs in the upper limb areas among the 3 patterns, indicating decreased lymphatic flow in moderate lymphedema. Interestingly, Dalia et al. [15] previously showed that both qualitative and quantitative lymphoscintigraphy were good methods for diagnosing lower limb lymphedema and were well correlated with each other. However, those authors included diseases such as deep vein thrombosis, venous insufficiency, erysipelas, trauma, and cancer. Unlike these previous studies, our study showed that quantitative lymphoscintigraphic analysis was well correlated with both qualitative lymphoscintigraphic analysis and the most commonly used measurement, circumference differences in the upper limbs, in breast cancer surgery patients with ALND.

Although this was a retrospective investigation, we also demonstrated the importance of quantitative lymphoscintigraphic analysis as an early diagnostic tool for lymphedema. Pecking et al. [19] revealed that qualitative data were always useful at the beginning of lymphatic dysfunction with no clinical edema apparent at the time of examination. Moreover, Dalia et al. [15] reported that quantitative interpretations are more sensitive than qualitative analyses for measuring circumference differences to differentiate normal patients from those with mild lymphedema. In this study, we included both proximal LNs and symptomatic upper limb areas in quantitative analysis for evaluating lymphatic flow in whole upper limbs. Additionally, our study used the MCDs in upper limbs, which were the same as the patients' uppermost symptomatic areas even when differences below $2 \mathrm{~cm}$ reflected high sensitivity in diagnosing lymphedema. An inter-limb difference of $2 \mathrm{~cm}$ is a widely used criterion for determining a positive test result, which leads to low sensitivity, which suggests that a negative result using this method alone may not provide sufficient information to rule out lymphedema [30]. Previous studies have emphasized that early clinical lymphedema detection and treatment are important for prognosis in breast cancer surgery patients with ALND [32-34]. Thus, quantitative lymphoscintigraphic analyses that use the QAI may be a new method for the early diagnosis of lymphedema severity for early treatment, even if patients do not yet show a large difference in the circumferences of their upper limbs.

Our study had some limitations. First, the number of patients with the qualitative normal pattern was small; 
this is because asymptomatic or early-stage lymphedema patients do not visit lymphedema clinics. Second, the included patients had different levels of ALND. Third, we could not analyze symptomatic hand swelling because the second interdigital space of the hand dorsum was the radiopharmaceutical injection site, and as such, radiation shielding with a lead board was placed on both hands. Fourth, we used only one type of radiopharmaceutical, ${ }^{99 \mathrm{~m}}$ Tc phytate, for economic reasons. Fifth, we measured only the circumference differences between upper limbs except for radiologic lymphoscintigraphic analysis. Finally, we do not yet have follow-up data. Future studies that include larger samples of patients, especially patients with the qualitative normal pattern, clearly stated levels of ALND, and long-term follow-up of both lymphoscintigraphy and clinical lymphedema severity as prognostic factors are needed to extend our results.

In conclusion, we found that the QAIs in the axillary LNs were lowest in the qualitative obstruction pattern, highest in the normal pattern, and inversely correlated with MCDs. In contrast, the QAIs in the symptomatic upper limbs were highest in the qualitative obstruction pattern and lowest in decreased function. These quantitative lymphoscintigraphic findings could well reflect both proximal and distal lymphatic flow in whole upper limbs. Thus, whenever we meet symptomatic patients after breast cancer surgery at any medical clinic, we should consider performing a quantitative lymphoscintigraphic analysis that comprehensively reflects both qualitative lymphatic dysfunction and circumference differences in the upper limbs for the early diagnosis of lymphedema and its severity in terms of lymphatic patency, so that early treatment can be recommended.

\section{CONFLICT OF INTEREST}

No potential conflict of interest relevant to this article was reported.

\section{REFERENCES}

1. Deo SV, Ray S, Rath GK, Shukla NK, Kar M, Asthana S, et al. Prevalence and risk factors for development of lymphedema following breast cancer treatment. Indian J Cancer 2004;41:8-12.

2. Warren AG, Brorson H, Borud LJ, Slavin SA. Lymph- edema: a comprehensive review. Ann Plast Surg 2007;59:464-72.

3. McLaughlin SA, Wright MJ, Morris KT, Giron GL, Sampson MR, Brockway JP, et al. Prevalence of lymphedema in women with breast cancer 5 years after sentinel lymph node biopsy or axillary dissection: objective measurements. J Clin Oncol 2008;26:5213-9.

4. Tiwari A, Cheng KS, Button M, Myint F, Hamilton G. Differential diagnosis, investigation, and current treatment of lower limb lymphedema. Arch Surg 2003; 138:152-61.

5. Bar Ad V, Cheville A, Solin LJ, Dutta P, Both S, Harris EE. Time course of mild arm lymphedema after breast conservation treatment for early-stage breast cancer. Int J Radiat Oncol Biol Phys 2010;76:85-90.

6. Hwang JM, Hwang JH, Kim TW, Lee SY, Chang HJ, Chu IH. Long-term effects of complex decongestive therapy in breast cancer patients with arm lymphedema after axillary dissection. Ann Rehabil Med 2013;37:690-7.

7. Johansson K, Holmstrom H, Nilsson I, Ingvar C, Albertsson M, Ekdahl C. Breast cancer patients' experiences of lymphoedema. Scand J Caring Sci 2003;17:3542.

8. Rietman JS, Dijkstra PU, Geertzen JH, Baas P, de Vries J, Dolsma WV, et al. Treatment-related upper limb morbidity 1 year after sentinel lymph node biopsy or axillary lymph node dissection for stage I or II breast cancer. Ann Surg Oncol 2004;11:1018-24.

9. Norman SA, Localio AR, Potashnik SL, Simoes Torpey HA, Kallan MJ, Weber AL, et al. Lymphedema in breast cancer survivors: incidence, degree, time course, treatment, and symptoms. J Clin Oncol 2009;27:390-7.

10. Deltombe T, Jamart J, Recloux S, Legrand C, Vandenbroeck N, Theys S, et al. Reliability and limits of agreement of circumferential, water displacement, and optoelectronic volumetry in the measurement of upper limb lymphedema. Lymphology 2007;40:26-34.

11. Szuba A, Shin WS, Strauss HW, Rockson S. The third circulation: radionuclide lymphoscintigraphy in the evaluation of lymphedema. J Nucl Med 2003;44:43-57.

12. Yuan Z, Chen L, Luo Q, Zhu J, Lu H, Zhu R. The role of radionuclide lymphoscintigraphy in extremity lymphedema. Ann Nucl Med 2006;20:341-4.

13. Sadeghi R, Kazemzadeh G, Keshtgar M. Diagnostic application of lymphoscintigraphy in the manage- 
ment of lymphoedema. Hell J Nucl Med 2010;13:6-10.

14. International Society of Lymphology. The diagnosis and treatment of peripheral lymphedema: Consensus document of the International Society of Lymphology. Lymphology 2003;36:84-91.

15. Dalia RM, Martins GR, Barbosa R, de Lima CF, Siqueira CF. Qualitative and quantitative lymphoscintigraphy in the evaluation of lower limbs lymphedema. Braz Arch Biol Technol 2005;48:159-62.

16. Hwang JH, Choi JY, Lee JY, Hyun SH, Choi Y, Choe YS, et al. Lymphscintigraphy predicts response to complex physical therapy in patients with early stage extremity lymphedema. Lymphology 2007;40:172-6.

17. Yoo J, Choi JY, Hwang JH, Kim DI, Kim YW, Choe YS, et al. Prognostic value of lymphoscintigraphy in patients with gynecological cancer-related lymphedema. J Surg Oncol 2014;109:760-3.

18. Stanton AW, Modi S, Mellor RH, Peters AM, Svensson WE, Levick JR, et al. A quantitative lymphoscintigraphic evaluation of lymphatic function in the swollen hands of women with lymphoedema following breast cancer treatment. Clin Sci (Lond) 2006;110:55361.

19. Pecking AP, Alberini JL, Wartski M, Edeline V, Cluzan RV. Relationship between lymphoscintigraphy and clinical findings in lower limb lymphedema (LO): toward a comprehensive staging. Lymphology 2008;41:1-10.

20. Francois A, Richaud C, Bouchet JY, Franco A, Comet $M$. Does medical treatment of lymphedema act by increasing lymph flow? Vasa 1989;18:281-6.

21. Franco A, Comet M, Beani JC, Doucet J, Meaulle PY, Guidicelli H. La lymphoscintigraphie indirecte: son intérêt pour le diagnostic étiologique des œdèmes des membres inférieurs de cause non générale. J Biophys Med Nucl 1980;4:299-303.

22. Szuba A, Strauss W, Sirsikar SP, Rockson SG. Quantitative radionuclide lymphoscintigraphy predicts outcome of manual lymphatic therapy in breast cancerrelated lymphedema of the upper extremity. Nucl Med Commun 2002;23:1171-5.

23. Kiel KD, Rademacker AW. Early-stage breast cancer: arm edema after wide excision and breast irradiation. Radiology 1996;198:279-83.
24. Keramopoulos A, Tsionou C, Minaretzis D, Michalas $\mathrm{S}$, Aravantinos D. Arm morbidity following treatment of breast cancer with total axillary dissection: a multivariated approach. Oncology 1993;50:445-9.

25. Piller N, Carati C. The diagnosis and treatment of peripheral lymphedema. Lymphology 2009;42:146-7.

26. Shaitelman SF, Cromwell KD, Rasmussen JC, Stout NL, Armer JM, Lasinski BB, et al. Recent progress in the treatment and prevention of cancer-related lymphedema. CA Cancer J Clin 2015;65:55-81.

27. Cheville AL, McGarvey CL, Petrek JA, Russo SA, Thiadens SR, Taylor ME. The grading of lymphedema in oncology clinical trials. Semin Radiat Oncol 2003;13:214-25.

28. Armer JM. The problem of post-breast cancer lymphedema: impact and measurement issues. Cancer Invest 2005;23:76-83.

29. Gebruers N, Truijen S, Engelborghs S, De Deyn PP. Volumetric evaluation of upper extremities in 250 healthy persons. Clin Physiol Funct Imaging 2007;27: 17-22.

30. Smoot BJ, Wong JF, Dodd MJ. Comparison of diagnostic accuracy of clinical measures of breast cancerrelated lymphedema: area under the curve. Arch Phys Med Rehabil 2011;92:603-10.

31. Pain SJ, Barber RW, Ballinger JR, Solanki CK, O'Mahony S, Mortimer PS, et al. Side-to-side symmetry of radioprotein transfer from tissue space to systemic vasculature following subcutaneous injection in normal subjects and patients with breast cancer. Eur J Nucl Med Mol Imaging 2003;30:657-61.

32. Bar Ad V, Dutta PR, Solin LJ, Hwang WT, Tan KS, Both S, Cheville A, Harris EE. Time-course of arm lymphedema and potential risk factors for progression of lymphedema after breast conservation treatment for early stage breast cancer. Breast J 2012;18:219-25.

33. Ramos SM, O'Donnell LS, Knight G. Edema volume, not timing, is the key to success in lymphedema treatment. Am J Surg 1999;178:311-5.

34. Forner-Cordero I, Munoz-Langa J, Forner-Cordero A, DeMiguel-Jimeno JM. Predictive factors of response to decongestive therapy in patients with breast-cancerrelated lymphedema. Ann Surg Oncol 2010;17:744-51. 